\title{
Factores psicosociales laborales y el Síndrome de Burnout en docentes de enseñanza media básica (secundaria) de la zona metropolitana de Guadalajara, México
}

\author{
MARÍA GUADALUPE ALDRETE ${ }^{(1)}$, JAQUELINE GONZÁLEZ ${ }^{(2)}$ y MARÍA DE LOURDES PRECIADO ${ }^{(1)}$
}

\section{RESUMEN}

Este estudio busca identificar la relación entre los factores psicosociales laborales y el Síndrome de Burnout en los docentes del nivel medio básico del sistema educativo oficial, de la Zona Metropolitana de Guadalajara, Jalisco, México.

Se realizó un estudio cuantitativo: descriptivo, transversal y observacional, en una muestra representativa de 360 maestros que laboraban frente a grupo, de una población de 8,935. Para la selección de los docentes se realizó un muestreo por conglomerados, seleccionando aleatoreamente 29 escuelas y de ellas a 13 docentes.

Para la captación de datos se utilizaron 3 instrumentos autoaplicados: Encuesta de datos generales y laborales, escala de Maslach Burnout Inventory (M.B.I:), Batería de Factores Psicosociales en el Trabajo Académico (FPSIS ACADEMICO).

Para el análisis y proceso de datos se utilizó el paquete estadístico Epi.Info 6.

Se encontró que el $80 \%$ de los docentes tenía alguna dimensión de la escala de Maslasch “quemada". Se identificó un $43.7 \%$ con agotamiento emocional, un $40.3 \%$ en baja realización personal, y un $13.3 \%$, con despersonalización. Los maestros identifican la presencia de factores de naturaleza psicosocial en los espacios laborales educativos, en las siete sub escalas que se evaluaron y como factor de riesgo para presentar agotamiento emocional, encontrando niveles altos con mayor frecuencia en: exigencias laborales $40.9 \%$ y remuneración del rendimiento $29.5 \%$.

Se concluye que la presencia de factores psicosociales laborales negativos en los espacios laborales afecta significativamente a los docentes, ocasionando un mayor riesgo de presentar agotamiento emocional.

Palabras clave: Síndrome de Burnout, agotamiento emocional, docentes, desgaste, salud ocupacional.

\begin{abstract}
PSYCHOSOCIAL OCCUPATIONAL FACTORS AND BURNOUT SYNDROME AMONG SECONDARY SCHOOL TEACHERS FROM THE METROPOLITAN ZONE OF GUADALAJARA, MÉXICO

The present study aims at identifying the link between psychosocial occupational factors and the Burnout Syndrome among high school teachers from the mainstream educative system at the

(1) Centro Universitario de Ciencias de la Salud. Departamento de Salud Pública. Instituto de Investigación en Salud Ocupacional. Universidad de Guadalajara. Menhir 1130. Fraccionamiento Colomos Altamira. Guadalajara. Jal. México.draaldrete@yahoo.com.mx

(2) Dirección de Higiene Escolar. Secretaría de Educación. Jal. México.
\end{abstract}




\section{Metropolitan Zone of Guadalajara, Jalisco, México.}

A quantitative descriptive, cross-sectional and observational study was carried out on a representative sample of 360 teachers who worked in front of a group, selected from a total population of 8,935. Cluster sampling was used for teacher selection: 29 schools were randomly selected and from these, 13 teachers were chosen.

Data collection used 3 self-applied instruments: a general and labor data survey, the Maslach Burnout Inventory (M.B.I) Scale, and a Psychosocial Factors in Academic Work assessment battery (ACADEMIC FPSIS).

The Epi.Info 6 statistic pack was used or data process and analysis.

The results showed that $80 \%$ of teachers had some dimension of the Maslach scale "burnt out". Emotional exhaustion was identified among 43.7\%, low personal accomplishment was observed in 40.3\%, and depersonalization in 13.3\%. Teachers evidenced the presence of psychosocial factors in the fields of educative work in the seven subscales that were evaluated, and as a risk factor for emotional exhaustion. High levels were most commonly observed among occupational demands $(40.9 \%)$ and performance compensation $29.5 \%$.

To conclude, the presence of negative labor psychosocial factors in occupational fields has a significant impact on teachers, resulting in a higher risk for emotional exhaustion.

Keywords: Burnout syndrome, emotional exhaustion, teachers, burnout, occupational health.

\section{INTRODUCCIÓN}

La secundaria empezó a considerarse parte de la educación básica mexicana en el Programa de Modernización Educativa durante el gobierno de Carlos Salinas de Gortari (1988-1994)' adquiriendo carácter legal en 1993, al modificarse el artículo tercero constitucional, en el que se define la educación básica como aquella integrada por los niveles de preescolar, primaria y secundaria, estableciendo un ciclo de educación básica obligatoria ${ }^{2}$. La secundaria se cursa en tres años, después de los seis de primaria, de esta forma la población escolar que acude a este nivel son adolescentes de 12 a 15 años.

Los fundamentos que dieron origen a esta reforma educativa están en relación a: aumentar la cobertura; adecuar la educación a las necesidades, intereses y capacidades de la población; disminuir la deserción; mejorar la equidad, y elevar la calidad de vida, entre otros ${ }^{3}$.

Para lograr esto la Secretaría de Educación Pública (SEP) ha tomado medidas tendientes a dotar de sentido a la secundaria en su nuevo marco de educación básica. Entre ellas la reforma de los planes y programas de estudio y el reconocimiento de la necesidad de la participación de los maestros en la comprensión, apropiación y compromiso con las nuevas orientaciones.

Sin embargo, la necesidad de implementar una reforma en un nivel educativo tan complejo, ha llevado a que se deje de lado la consideración de que al promover un solo ciclo básico, todos sus maestros son iguales y enfrentan las mismas condiciones; situación que está muy lejos de la realidad, ya que los profesores de secundaria son distintos a los de primaria, no solamente por su formación profesional, sino también por sus expectativas profesionales y condiciones laborales. Esto ha traído como consecuencia la existencia de un sinnúmero de agentes de naturaleza psicosocial, que se consideran como estresores, y que la Organización Internacional del Trabajo (OIT), en 1984, definió como elementos externos que afectan las relaciones de la persona con su grupo y cuya presencia puede producir daño en el equilibrio psicológico del individuo ${ }^{4}$. Es por ello que esta misma institución habla de factores de riesgo psicosocial como las interacciones entre el contenido, la organización y la gestión del trabajo y las condiciones ambientales por un lado y las capacidades del trabajador, sus funciones y necesidades por otro ${ }^{5}$.

El docente de enseñanza media básica debe 
tener una serie de habilidades y actitudes que le permitan proyectar y poner en práctica las estrategias más adecuadas para promover el aprendizaje y la construcción de habilidades actitudes y saberes en los educandos, más allá de la mera instrucción trasmisora de conocimientos propios de un sistema de enseñanza tradicional.

Este rol requiere de profesionales comprometidos social e institucionalmente, que sean capaces de intervenir adecuadamente ante un alumno en etapa de crisis como es la adolescencia y en los que muchas veces el aprender no está dentro de sus prioridades. Por otra parte, la docencia es considerada como una profesión de servicio y de entrega, en la cual se propicia la vinculación emocional personal y donde se involucra la capacidad afectiva de las personas, por esta razón es posible que algunos docentes desarrollen el Síndrome de Burnout.

Hablar del Síndrome de Burnout, de agotamiento profesional o de "Quemarse" por el trabajo y las cuestiones relacionadas con éste, son un tema en boga. Su estudio ha sido abordado desde el primer tercio del siglo XX, cuando se comenzaron a realizar investigaciones para encontrar los distintos factores que lo propician.

A partir de los 80's se iniciaron las investigaciones centradas en el estrés docente y más específicamente en el Síndrome de Burnout, entendido como un trastorno de la adaptación que se presenta por un estrés crónico y puede desencadenar diversos síntomas tanto físicos como psicológicos, los que dañan significativamente la ejecución profesional y afectan las relaciones mantenidas con los alumnos y la calidad de la enseñanza.

Algunos estudios recientes en México sobre el Síndrome de Burnout en los maestros de enseñanza básica, han encontrado que este síndrome está relacionado con la antigüedad (a mayor antigüedad mayor agotamiento emocional); con el nivel de escolaridad (a mayor escolaridad más probabilidad de quemarse, porque el docente percibe que tendría más probabilidades de promoción), y con la falta de apoyo familiar (el $80 \%$ son maestras que tienen que realizar doble jornada, en la escuela y en el hogar $)^{6}$.

Salgado ${ }^{7}$ argumenta que es más frecuente el síndrome en docentes de secundaria que en los maestros de primaria, tal vez por la etapa de adolescencia que viven los alumnos y que demanda mayor vinculación afectiva, la naturaleza institucional de la escuela secundaria, el conflicto de roles profesor-alumno entre otros y Sevilla en 2002, encontró que los perfiles académicos de formación del docente de secundaria no coinciden con los de las materias bajo su responsabilidad e imparte materias de distintas áreas ${ }^{8}$

De aquí que el objetivo del presente trabajo fue identificar la presencia factores psicosociales laborales y su relación con el Síndrome de Burnout, de los docentes de Educación Media Básica (secundaria) de la Zona Metropolitana de Guadalajara, Jalisco, México.

\section{MATERIAL Y MÉTODO}

Se realizó un estudio descriptivo, observacional y analítico, en una muestra 360 maestros, de 29 escuelas secundarias de la Zona Metropolitana de Guadalajara integrada por los municipios de Guadalajara, Zapopan, Tlaquepaque y Tonalá. Se realizó un muestreo por conglomerados y a través de un muestreo aleatorio simple se seleccionaron las escuelas y, de éstas, a 13 docentes.

Las unidades de observación fueron seleccionadas de acuerdo a los siguientes criterios: maestros con más de 6 meses de laborar frente a grupo, presentes el día que se visitó la escuela, y con una carga horaria de más de 15 horas semanales en la escuela seleccionada. Se excluyeron maestros que se negaron a contestar el formulario, maestros ausentes por permiso o incapacidad y que realizaran funciones administrativas exclusivamente.

La información fue obtenida mediante un formulario estructurado que incluyó cuestionario de datos generales y laborales de los maestros: edad, sexo, escolaridad, antigüedad, turno de trabajo, horas frente a grupo, actividad como docente en otro centro escolar y subsistema al pertenece la escuela. Un segundo instrumento 
fue enfocado al rescate de los indicadores del Síndrome de Burnout a través del Maslach Burnout Inventory (M.B.I.), se utilizó una versión traducida y validada9 ${ }^{9}$ Es un instrumento que consta de 22 ítems con respuestas de opción múltiple, tipo Likert, que permite identificar el agotamiento emocional, la baja realización personal y despersonalización. Las puntuaciones de cada escala se obtienen al sumar los valores identificados para cada una de las dimensiones. Como puntos de corte se utilizó la versión Catalana del MBI, que clasifica en nivel "alto", "medio" y "bajo o nulo" cada una de las tres subescalas del instrumento, considerando como "quemada" cada dimensión que se registre en las calificaciones de nivel alto como las de nivel medio. Este instrumento tiene una confiabilidad para agotamiento emocional de una alfa de Cronbach de 0.90 , una alfa de 0.76 para despersonalización y una alfa de 0.76 para realización personal en el trabajo.

Un tercer instrumento fue la escala de Factores Psicosociales en el Trabajo Académico de Silva Gutiérrez. Éste se estructura en 7 subescalas: condiciones del lugar de trabajo; carga de trabajo; contenido y características de la tarea; exigencias laborales; papel del académico y desarrollo de la carrera; interacción social y aspectos organizacionales, y remuneración del rendimiento. Este instrumento utiliza una escala de frecuencia de 5 grados, tipo Likert, que va desde nunca hasta siempre. Para su evaluación se suman los puntajes de cada apartado y se clasifican en tres categorías: bajo, medio y alto $^{10}$.

El procesamiento de los datos se realizó en el programa EPI-INFO 6. Para el análisis se utilizó estadística descriptiva y pruebas paramétricas de acuerdo a la naturaleza de las variables. La asociación entre variables se determinó a través de chi-cuadrada, con un valor de significancia de $\mathrm{p}=<0.05$, un O.R. mayor que 1 y que el intervalo de confianza no incluyera la unidad.

Al maestro se le informó que su participación era voluntaria y se consideró el consentimiento informado de tipo verbal y personal.

\section{RESULTADOS}

La población estudiada estuvo constituida por 360 docentes, de los cuales el $53.8 \%$ eran mujeres y $46.2 \%$ hombres; $64.5 \%$ estaba casado; el $20.7 \%$ era soltero, y el $14.8 \%$ tenía otra situación legal. La edad de los participantes fluctuó de 23 a 65 años con un promedio de 43 $( \pm 8.6)$ años.

Respecto a la escolaridad de los docentes, el $82.5 \%$ posee licenciatura, $15.8 \%$ ha cursado posgrado a nivel maestría, y $1.6 \%$ tiene carrera técnica.

En relación con la formación en materia educativa, el 51.6\% de los maestros cursó una licenciatura relacionada con educación, y el $48.4 \%$ lo hizo en licenciaturas no relacionadas con la educación, siendo las más frecuentes del área de ciencias exactas (ingeniería), sociales (derecho) y salud (medicina).

El 50.6\% de los docentes de la escuela donde fueron captados pertenece al subsistema estatal y el $49.4 \%$ al subsistema federalizado (integrado).

El $87.7 \%$ posee un nombramiento como docente y el resto también tiene otro administrativo. La antigüedad en la docencia fluctuó entre 1 y 36 años, con un promedio de 17.8 años $( \pm 7.3)$.

El $48.9 \%$ de los docentes laboraba en el turno mixto, es decir tienen algunas horas por la mañana y otras por la tarde. El número de alumnos que atiende fluctuó desde 22 hasta 700, el promedio fue de $149( \pm 103)$.

Los maestros del estudio imparten un promedio de 30.3 horas a la semana ( \pm 10.5$)$, frente a grupo en la escuela donde fueron captados y el $29.4 \%$ tiene en promedio 16.7 horas frente a grupo en otro centro educativo, en el mismo nivel de educación. $23 \%$ realiza docencia en otro nivel educativo y $24.4 \%$ tiene otro empleo no relacionado con docencia.

En relación al Síndrome de Burnout, se encontró que sólo el 19.2\% no lo presentaba. Esto es ninguna dimensión "quemada", lo que quiere decir que ocho de cada 10 docentes tienen al menos una dimensión afectada o "quemada". Para esto se consideró la calificación como alta 
y media en cada una de las dimensiones $(46.1 \%$ tenían una dimensión "quemada", $17.2 \%$ tenían 2 y $17.5 \%$ las tres dimensiones quemadas).

Se identificó un $43.7 \%$ con agotamiento emocional, un $40.3 \%$ en baja realización personal y un $13.3 \%$, con despersonalización. Esto es sumando los porcentajes obtenidos en la calificación como alto y medio.

Los maestros identifican la presencia de factores de naturaleza psicosocial en los espacios laborales educativos, en las siete subescalas de estos factores, encontrando niveles altos con mayor frecuencia en: exigencias laborales $40.9 \%$, remuneración del rendimiento $29.5 \%$, papel del académico y desarrollo de la tarea (Tabla 1).

Para encontrar la relación entre los factores psicosociales y el Síndrome de Burnout, se agruparon los resultados obtenidos en ambas escalas en alto y medio, contra el resultado de bajo encontrando relación con agotamiento emocional y los factores psicosociales en las 7 subescalas que se evaluaron (condiciones del lugar de trabajo, carga de trabajo, contenido y características de la tarea, exigencias laborales, papel del académico y desarrollo de la carrera, interacción social y aspectos organizacionales, remuneración del rendimiento). Así como los factores de las condiciones de trabajo, contenido y características de la tarea y de la interacción social y aspectos organizacionales con la dimensión de despersonalización y con baja realización personal, sólo se encontró relación con la interacción social y aspectos organizacionales (Tabla 2).

Se analizaron otras variables y se encontró como factor de riesgo para desarrollar el Síndrome de Burnout la formación académica diferente al área pedagógica ( $\mathrm{p}=.000$ O.R. 5.54 I.C. 3.89-7.92) y haber realizado estudios de maestría se relacionó con la baja realización personal $(\mathrm{P}=.000$, O.R. 3.82 I.C. 2.65-5.52).

\section{DISCUSIÓN}

Este estudio tenía como objetivo principal detectar la presencia del Síndrome de Burnout en docentes y su relación con los factores psicosociales.

La cifra de prevalencia de $80 \%$ es -sin dudaun hallazgo importante y preocupante, ya que 8 de cada 10 presenta este problema. Se encontraron datos mayores que lo reportado por Unda $^{11}$ 2007, en profesores del Colegio de Ciencias (60\%); por Pando ${ }^{12}$ en 2006, en docentes universitarios (38.9\%); por Rocha ${ }^{13}$, 2006 en profesores de bachillerato (43.3\%), y Restrepo ${ }^{14}$ en docentes nivel básico (23.4\%.) y datos semejantes a la prevalencia reportada por Aldrete $^{6}, 2003$ en docentes de nivel primaria, y Piñeyro ${ }^{15}$ en nivel secundaria. Esto nos lleva a pensar que el trabajo que realiza el docente del nivel primaria y secundaria, es más demandante.

Tabla 1. Áreas de los factores psicosociales del trabajo académico en los docentes de educación media básica de la ZMG

\begin{tabular}{|c|c|c|c|c|c|c|}
\hline \multirow{3}{*}{ Áreas de los factores psicosociales } & \multicolumn{6}{|c|}{ NIVELES } \\
\hline & \multicolumn{2}{|c|}{ Alto } & \multicolumn{2}{|c|}{ Medio } & \multicolumn{2}{|c|}{ Bajo } \\
\hline & $\mathrm{Fc}$ & $\%$ & $\mathrm{Fc}$ & $\%$ & $\mathrm{Fc}$ & $\%$ \\
\hline 1.Condiciones del lugar de trabajo & 37 & $10 . \%$ & 236 & $65.6 \%$ & 87 & $24.2 \%$ \\
\hline 2.Carga de trabajo & 75 & $20.8 \%$ & 239 & $66.4 \%$ & 46 & $12.8 \%$ \\
\hline 3.Contenido y características de la tarea & 18 & $5 \%$ & 259 & $71.9 \%$ & 83 & 23.1 \\
\hline 4.Exigencias laborales & 147 & $40.9 \%$ & 183 & $51.0 \%$ & 29 & $8.1 \%$ \\
\hline 5.Papel del académico y desarrollo de la tarea & 77 & $21.4 \%$ & 224 & $62.4 \%$ & 58 & $16.2 \%$ \\
\hline 6.Interacción social y aspectos organizativos & 42 & 11.7 & 163 & 45.4 & 154 & 42.9 \\
\hline 7.Remuneración del rendimiento & 106 & $29.5 \%$ & 128 & $35.7 \%$ & 125 & $34.8 \%$ \\
\hline
\end{tabular}


Tabla 2. Valores significativos entre las áreas de los factores psicosociales y las dimensiones del Síndrome de Burnout

\begin{tabular}{|c|c|c|c|c|}
\hline \multirow{2}{*}{$\begin{array}{c}\text { Batería de factores } \\
\text { psicosociales } \\
\text { en el trabajo académico }\end{array}$} & \multicolumn{4}{|c|}{$\begin{array}{l}\text { Dimensiones del Síndrome de Burnout } \\
\text { Escala de M.B.I. }\end{array}$} \\
\hline & $\begin{array}{l}\text { Agotamiento } \\
\text { emocional }\end{array}$ & Despersonalización & $\begin{array}{l}\text { Baja realización } \\
\text { personal }\end{array}$ & $\begin{array}{c}\text { Más de una } \\
\text { dimensión quemada }\end{array}$ \\
\hline Condiciones del lugar de trabajo & $\begin{array}{l}2.89(1.63-5.17) \\
\mathrm{p}=0.0000^{*}\end{array}$ & $\begin{array}{l}5.53(1.58-23.17) \\
\quad p=0.0018^{*}\end{array}$ & $\begin{array}{c}148(.86-2.54) \\
\mathrm{p}=0.129\end{array}$ & $\begin{array}{l}2.10(1.14-3.85) \\
\mathrm{p}=0.0092 *\end{array}$ \\
\hline Carga de trabajo & $\begin{array}{c}2.7(1.29-6.06) \\
p=0.003^{*}\end{array}$ & $\begin{array}{c}1.03(.38-2.91) \\
\mathrm{p}=0.950\end{array}$ & $\begin{array}{c}.78(.40-1.52) \\
\mathrm{p}=0.426\end{array}$ & $\begin{array}{c}.97(.42-2.31) \\
\mathrm{p}=0.94\end{array}$ \\
\hline $\begin{array}{l}\text { Contenido y características } \\
\text { de la tarea }\end{array}$ & $\begin{array}{c}2.64(1.48-4.73) \\
p=0.000^{*}\end{array}$ & $\begin{array}{c}2.87(1.03-8.62) \\
\mathrm{p}=0.0255^{*}\end{array}$ & $\begin{array}{c}1.44(.83-2.49) \\
\mathrm{p}=0.16\end{array}$ & $\begin{array}{c}1.93(1.03-3.57) \\
\mathrm{p}=0.0241^{*}\end{array}$ \\
\hline Exigencias laborales & $\begin{array}{c}5.40(1.72-18.75) \\
p=0.000^{*}\end{array}$ & $\begin{array}{c}.96(.30-3.47) \\
\mathrm{p}=0.944\end{array}$ & $\begin{array}{c}.96(.41-2.23) \\
\mathrm{p}=0.909\end{array}$ & $\begin{array}{c}1.53(.48-5.45) \\
\mathrm{p}=0.43\end{array}$ \\
\hline $\begin{array}{l}\text { Papel de académico y desarrollo } \\
\text { de la carrera }\end{array}$ & $\begin{array}{c}3.17(1.57-6.52) \\
p=0.000^{*}\end{array}$ & $\begin{array}{c}2.31(.75-8.00) \\
\mathrm{p}=0.113\end{array}$ & $\begin{array}{c}1.35(.83-2.44) \\
\mathrm{p}=0.316\end{array}$ & $\begin{array}{c}.63(.31-1.28) \\
\mathrm{p}=0.160\end{array}$ \\
\hline $\begin{array}{l}\text { Interacción social y aspectos } \\
\text { organizacionales }\end{array}$ & $\begin{array}{c}2.77(1.73-4.44) \\
\mathrm{p}=0.0000^{*}\end{array}$ & $\begin{array}{c}1.99(.98-4.09) \\
\mathrm{p}=0.0389^{*}\end{array}$ & $\begin{array}{c}1.63(1.03-2.58) \\
\mathrm{p}=0.0266^{*}\end{array}$ & $\begin{array}{c}3.97(2.17-7.31) \\
\mathrm{p}=0.0000^{*}\end{array}$ \\
\hline Remuneración del rendimiento & $\begin{array}{c}1.72(1.07-2.77) \\
\mathrm{p}=0.017^{*}\end{array}$ & $\begin{array}{c}.87(.44-1.73) \\
p=0.675\end{array}$ & $\begin{array}{c}1.26(.78-2.03) \\
\mathrm{p}=0.310\end{array}$ & $\begin{array}{c}.86(.48-1.54) \\
\mathrm{p}=0.57\end{array}$ \\
\hline Todos los factores & $\begin{array}{c}5.65(2.45-13.52) \\
\mathrm{p}=0.0000^{*}\end{array}$ & $\begin{array}{c}2.16(.69-1.48) \\
\mathrm{p}=0.148\end{array}$ & $\begin{array}{l}1.12(.59-211) \\
\mathrm{p}=0.716\end{array}$ & $\begin{array}{c}2.97(1.50-5.87) \\
\mathrm{p}=0.0004^{*}\end{array}$ \\
\hline
\end{tabular}

* Valores significativos.

Las asociaciones que se mostraron en los resultados entre las dimensiones del Síndrome y los factores laborales negativos en el trabajo académico, demuestran que si existe relación entre la presencia de los factores psicosociales laborales y el Síndrome de Burnout, evidencian que los docentes en los centros escolares no sólo ponen de manifiesto sus conocimientos y estrategias de aprendizaje, sino básicamente sus habilidades y recursos para relacionarse con los alumnos, compañeros, padres de familia, fun- cionarios de la organización, administración institucional, familia etc, lo que le exige un esfuerzo psíquico que puede propiciar que se desarrolle el Síndrome de Burnout, coincidiendo con lo que argumenta Guerra ${ }^{16}$.

Según el modelo teórico de Maslach, el Síndrome de Burnout es un proceso en que el agotamiento emocional es la dimensión precursora del Síndrome, siguiéndose por despersonalización y, finalmente, el sentimiento de disminución del logro personal en el trabajo ${ }^{17}$. En el 
presente estudio se visualiza el agotamiento emocional como la dimensión en la cual casi la mitad de los docentes presentaron mayor problema.

El estudio indica que el $29.4 \%$ de los docentes labora en más de un plantel educativo, datos semejantes fueron reportados por Sevilla ${ }^{8}$ donde el $28 \%$ de profesores de secundarias viven este problema, dificultando el sentido de pertenencia a su escuela.

El contenido y características de la tarea que el docente realiza, lo llevan a agotarse emocionalmente. El docente reporta exigencias laborales problemas con la interacción social, con las condiciones de trabajo, enfrenta problemas con el comportamiento de sus alumnos, la demanda de atención por parte de los mismos y la cantidad de alumnos que atiende. En la Reforma de Educación Secundaria se estimó que en las zonas urbanas, los profesores que imparten asignaturas de tres horas por semana atienden doce grupos y alrededor de 600 alumnos, para cubrir sus horas de nombramiento, situación que vivencian los docentes de este estudio ${ }^{18}$, aunado a todo esto que muchos de ellos no tienen la formación pedagógica.

\section{CONCLUSIONES}

Las condiciones laborales que los docentes de secundaria viven cotidianamente los están llevando a agotarse emocionalmente y a despersonalizarse, como lo reflejan las cifras de prevalencia del Síndrome de Burnout encontradas por el estudio.

Resulta necesaria la intervención asistencial en los casos ya detectados y realizar estrategias de prevención en los docentes que se encuentran en riesgo de padecer el Síndrome de Burnout.

Resulta conveniente mejorar los espacios y las condiciones laborales docentes, así como implementar estrategias de mejoramiento de las relaciones humanas que potencialicen habilidades sociales.

Recepción: 16 de mayo de 2008 Aprobación: 17 de julio de 2008

\section{REFERENCIAS}

1. SANDOVAL FLORES E. Ser maestro de secundaria en México: Condiciones de trabajo y reformas educativas - , enero - abril 2001, http://www.rieoei.org/rie25a04.htm

2. LEY GENERAL DE EDUCACIÓN, CÁMARA DE DIPUTADOS DEL H. CONGRESO DE LA UNIÓN. Ultima reforma DOF 22-06-2006 en: www.ei.es/quipu/mexico/ley_gen_educ.pdf

3. ZORRILLA M. La educación secundaria en México: al filo de la reforma, REICE Revista Electrónica Iberoamericana sobre Calidad, Eficiencia y Cambio en Educación, 2004 Vol. 12 No. 1 www.ice.deusto.es/rinace/reice/vol12n1.

4. ORGANIZACIÓN INTERNACIONAL DEL TRABAJO, OIT. Factores psicosociales en el trabajo: naturaleza, incidencia y prevención. Ginebra. Informe del Comité Mixto OIT/OMS, Ginebra 18-24 de septiembre de 1984 www.metabase.net/docs/oit/02294.html

5. VELÁZQUEZ M. Los riesgos psicosociales en el Trabajo, 2003, Disponible en:

www.arearh.com/alud\%20laboral/psicosociales2.htm

6. ALDRETE, R. M. G., PANDO, M. M., ARANDA, B. C., BALCÁZAR, P. N. Síndrome de Burnout en maestros de educación básica de Guadalajara. Revista de Investigación y Salud, Vol. V, Número 1, abril, 2003:1116.

7. SALGADO RUIZ. A. El Síndrome de "Burnout": estudio empírico en profesores de enseñanza primaria. Universidad Pontificia de Salamanca. España. Anales Revista Psicología General y Aplicada. Iberpsicología, 1999, 21.

8. SEVILLA MORENO; U. Y VILLANUEVA VELASCO R. La salud laboral docente en la enseñanza pública gabinetes de estudios y de salud laboral. Publicaciones de la Federación de enseñanza de comisiones obreras. Acción Sindical Federación de enseñanza, Vol. 2, España, Abril, 2002.

www.Fe.eccoo.es/pdf/n%200\%20salud\%20laboral. pdf

9. GIL-MONTE, P.R. Validez factorial de la adaptación al español del Maslach Bumout Inventory-General Survery. Salud Pública de México; 2002;44(2):33-40

10. SILVA GUTIÉRREZ N. Factores psicosociales, estrés y desgaste profesional en académicos del Centro Universitario de Ciencias Biológicas Agropecuarias de la Universidad de Guadalajara, Tesis del Doctorado en Ciencias de la Salud en el Trabajo de la Universidad de Guadalajara, 2006, México.

11. UNDA ROJAS S. SANDOVAL OCAÑA J., Síndrome de desgaste profesional o burnout en el colegio de ciencias sociales y humanidades, http://www.unem.mx/foroamericasmemorias/ponen cias/pdf/p4\%29SUnda

12. PANDO MORENO M., Factores psicosociales y 
Síndrome de Burnout en docentes de la Universidad del Valle de Atemajac, Guadalajara, México. Revista Salud en Tabasco 2006, Vol 12 No. 3: 523-529.

13. ROCHA ARANDA L., Prevalencia del Síndrome de Burnout en profesores de bachillerato de la Universidad de Guadalajara, Revista Humanitatis, 2006, Año III No. 9 :63-67.

14. RESTREPO AYALA, N.C., COLORADO VARGAS, G.O., CABRERA ARANA, G. Desgaste profesional en docentes oficiales de Medellín, Colombia, 2005. Rev. salud pública vol.8 no.1 Bogotá ,2006:63-73.

15. PIÑEYRO, NEREIDA Y COLS. Estudio sobre la presencia del Síndrome de Burnout en los profesores de la secundaria básica José Martí del municipio del Cotorro, Revista Ciencias. Código ISPN de la Publicación: EEuAlyAAEAwLXVikpV Cuba, 2006.

16. GUERRA, CID LUIS RAIMUNDO, JIMÉNEZ, TORRES SILVIA. El malestar docente: un estudio empírico y comparativo localizado en: Psiquiatría.com, www.intersalud.es. 2004.

17. CARLOTTO, MS., PALAZZO, L. Factors associated with Burnout's Syndrome: an epidemiological study of teachers. Cad. Saúde Pública, 2006;22(5):10171026.

18. SECRETARÍA DE EDUCACION PUBLICA, Reforma de la educación secundaria, SEP, 2002.

Usted puede comentar éste y otros artículos publicados en la Revista Chilena de Salud Pública, enviando un correo electrónico a revistasp@ med.uchile.cl 